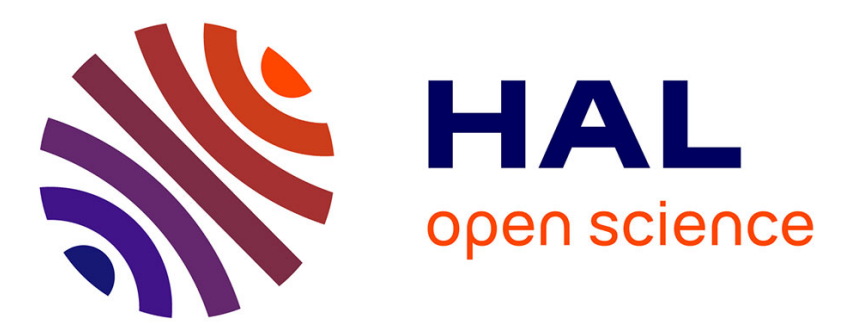

\title{
L'identité numérique du locuteur dans les échanges professionnels en ligne: le cas du forum médical infirmiers.com
}

Hani Qotb

\section{- To cite this version:}

Hani Qotb. L'identité numérique du locuteur dans les échanges professionnels en ligne: le cas du forum médical infirmiers.com. Langage et Société, 2020, Varia, 170 (2), pp.129-146. 10.3917/ls.170.0129 . hal-02940231

\section{HAL Id: hal-02940231 \\ https://hal.univ-lorraine.fr/hal-02940231}

Submitted on 16 Sep 2020

HAL is a multi-disciplinary open access archive for the deposit and dissemination of scientific research documents, whether they are published or not. The documents may come from teaching and research institutions in France or abroad, or from public or private research centers.
L'archive ouverte pluridisciplinaire HAL, est destinée au dépôt et à la diffusion de documents scientifiques de niveau recherche, publiés ou non, émanant des établissements d'enseignement et de recherche français ou étrangers, des laboratoires publics ou privés. 


\title{
L'identité numérique du locuteur dans les échanges professionnels en ligne : le cas du forum médical infirmiers.com
}

\author{
Hani Qotb \\ ATILF / UMR 7118-CNRS \\ haniqotbdyahoo.fr
}

\section{Introduction}

Grâce à l'omniprésence des technologies de l'information et de la communication (TIC), les individus sont de plus en plus amenés à s'exprimer en ligne sur les divers thèmes de leur vie sociale ou professionnelle. Cette tendance a contribué à l'émergence du web social qui permet à l'internaute d'être désormais au centre du processus de production et de diffusion des contenus en ligne. Ce phénomène numérique ne fait qu'affirmer la culture participative du web marquée par la collaboration dans la mesure où l'idée du partage est le fondement des activités sur les réseaux sociaux (Breslin, Passant \& Decker 2009). Dans ce contexte, le développement des activités numériques (diffuser et commenter du contenu, proposer des idées, échanger des ressources, exprimer des points de vue, etc.) a favorisé la présence des formes discursives numériques conçues comme "des productions verbales élaborées en ligne, quels que soient les appareils, les interfaces, les plateformes ou les outils d'écriture " (Paveau 2017 : 8). Ainsi, l'Analyse du Discours Numérique $(\mathrm{ADN})$ ne se limite plus aux aspects langagiers mais ces derniers se trouvent dorénavant indissociablement liés aux aspects techniques permettant de rendre la production des échanges en ligne plus riches aux niveaux informationnel et formel. Cet article a pour objectif d'étudier les spécificités de l'identité numérique notamment ses dimensions sociale et

(C) Langage \& Société $\mathrm{n}^{\circ} 170-2020 / 2$ 
discursive chez les locuteurs professionnels. Pour ce faire, nous analyserons la construction identitaire dans un environnement technodiscursif, l'impact des réseaux sociaux sur l'identité numérique professionnelle, les procédés adoptés par le locuteur et la part du social dans le discours numérique produit dans un réseau spécialisé. Ces éléments de recherche constituent le fil conducteur de cette étude sur l'identité numérique du locuteur dans le monde professionnel.

\section{Les spécificités de l'identité du locuteur}

Le terme d'identité trouve ses origines en latin "identitas " qui signifie le même. Selon le Trésor de la langue française (TLF), l'identité constitue : "L'ensemble des traits ou caractéristiques qui, au regard de l'état civil, permettent de reconnaître une personne et d'établir son individualité au regard de la loi. " Cette définition s'intéresse aux aspects individuels (nom, prénom, âge, etc.) qui contribuent à distinguer une personne par rapport à la vie civile. Selon Charaudeau (2009 : 340), l'identité donne la possibilité au sujet de prendre conscience de son existence, son corps, son savoir, ses jugements et ses actions. Le même auteur (Charaudeau 2009: 347) met l'accent sur deux dimensions principales de l'identité du locuteur. D'une part, l'identité sociale a la particularité d'être reconnue par les autres dans une situation de communication donnée dans la mesure où le locuteur est amené à répondre à la question suivante : "Je suis là pour quoi dire, en fonction du statut et du rôle qui m’est assigné par la situation. " D'autre part, l'identité discursive se caractérise par le fait qu'elle est construite par le sujet-parlant lui-même pour répondre à la question suivante : "Je suis là pour comment parler ? " Elle sert à refléter et affirmer l'identité sociale dans les différents échanges qu'ils soient en présentiel ou en ligne. D'où les relations étroites, voire ce " jeu " à la fois construit et négocié grâce aux dimensions sociale et discursive qui contribuent à la construction identitaire du sujet-parlant. En fait, l'identité discursive se compose de deux notions principales à savoir l'ethos et le pathos. D'un côté, l'ethos renvoie à la représentation de soi d'un sujet-parlant ou pensant dans son discours. Cette notion peut être définie comme «l'image de soi que le locuteur construit dans son discours pour exercer une influence sur son allocutaire" (Amossy 2002 : 238). Pour sa part, Maingueneau (2002 : 81) met en lumière deux aspects de l'ethos: l'ethos dit verbal et l'ethos montré. Le premier représente "les fragments du texte où l'énonciateur évoque sa propre énonciation ". Quant à l'ethos montré, il se manifeste à travers les comportements du locuteur (débit, intonation, arguments, choix des mots, etc.). D'un 
autre côté, le pathos a pour but de provoquer des réactions (verbales ou physiques), des émotions et des passions chez l'auditoire visé. Dans ce contexte, Charaudeau (2005) souligne deux caractéristiques principales de l'identité discursive : la crédibilité et la captation. Celle-là est liée au besoin du sujet-parlant d'être cru par son entourage et d'être pris au sérieux. Concernant la captation, elle se manifeste en absence d'une relation d'autorité avec l'interlocuteur. Elle consiste à amener ce dernier à partager ses idées et ses opinions en adoptant trois attitudes discursives principales à savoir : la polémique (mettre en cause les idées de son adversaire), la séduction (présenter un imaginaire dont l'interlocuteur peut bénéficier) et la dramatisation (décrire des faits de la vie à travers des analogies, des comparaisons, des métaphores, etc.). Ces stratégies discursives ont le mérite de rendre le discours du sujet-parlant à la fois crédible et attractif auprès de ses interlocuteurs.

\section{Le forum comme dispositif technodiscursif en ligne}

Dans le cadre de notre étude, nous nous intéressons au forum conçu comme " un service permettant l'échange et la discussion en ligne sur un thème donné » (Le Journal Officiel 1999). Chaque utilisateur peut lire à tout moment les interventions de tous les autres et apporter sa propre contribution sous forme d'articles. De manière générale, les forums sont marqués par leur dimension quadruple (Mangenot 2002) : écrite, asynchrone, publique et structurée. D'abord, il s'agit d'échanges qui se déroulent par écrit sans utiliser les autres modes de communication audio ou audiovisuelle. Pourtant, nous constatons parfois la présence des schémas ou des émojis qui reflètent l'oralité dans les échanges écrits. Quant à l'aspect asynchrone, il porte sur une communication qui se passe en temps différé, donnant ainsi une souplesse temporelle aux participants selon leur disponibilité. De même, le forum est public (ou semi-public) en opposition aux discussions privées. Enfin, il y a l'aspect structuré qui différencie forum et liste de diffusion. Pour sa part, Bruillard (2006) ajoute l'aspect collectif ou polylogue du forum où il faut la participation de plusieurs personnes en vue de mener le débat sur un thème donné.

Dans le cadre des échanges produits en ligne, de nombreux spécialistes (Iteanu 2008 ; Licoppe 2009 ; Georges 2009 ; Paveau 2012a) mettent en lumière la notion d'identité numérique qui correspond " aux traces de l'individu qui sont laissées sur des supports numériques » (Loisy, Mailles-Viard Metz \& Benech 2010 : 2). Selon Georges (2009), l'identité numérique est distinguée par trois dimensions principales : identité déclarative (données personnelles fournies par l'internaute : 
nom, prénom, âge, profession, etc.), identité calculée (nombre d'amis, de photos, etc.) et identité agissante (lancer un événement, proposer des idées, demander de l'aide, etc.). En fait, l'identité déclarative et l'identité calculée permettent de constituer l'identité sociale du locuteur qui détermine en grande partie son identité discursive et ses échanges avec ses interlocuteurs sur Internet. C'est pourquoi il s'avère important d'analyser les deux facettes de l'identité (sociale et discursive) du locuteur dans un forum professionnel.

\section{Le corpus d'étude et la méthodologie de recherche}

Chaque domaine professionnel a tendance à développer sa présence sur Internet. C'est le cas, par exemple, du monde médical qui est omniprésent sur la Toile sous plusieurs formes numériques : sites institutionnels (ministère des Solidarités et de la Santé ${ }^{1}$, Santé publique France ${ }^{2}$, Assurance maladie $e^{3}$, etc.), Facebook (Médecins sans frontières ${ }^{4}$, Médecins de France 5 , etc.), Twitter (Ordre des médecins, Le Quotidien du médecin, Médecins du monde, etc.), blogs (Le club des médecins blogueurs ${ }^{6}$, Armance', etc.) et Youtube (La chaine des aides-soignantes ${ }^{8}$, Fréquence médicale ${ }^{9}$, Aviscène ${ }^{10}$, etc.). Ces différents réseaux institutionnels et sociaux ont pour but de favoriser les échanges entre les professionnels du monde médical et mutualiser leurs connaissances et leurs expériences. Parmi ces réseaux numériques, nous nous sommes intéressés au site infirmiers. com $^{11}$ qui est marqué par la présence de six rubriques thématiques : concours, étudiants, profession, spécialités, exercice libéral et métiers du soin. Notre choix est dû à plusieurs raisons. D'abord, ce site fait partie du Groupe Profession Santé $e^{12}$ comprenant d'autres sites professionnels (Le Quotidien du Médecin ${ }^{13}$, Le Quotidien du

1. Voir en ligne $:<$ https://solidarites-sante.gouv.fr/>.

2. Voir en ligne $:<$ http://www.santepubliquefrance.fr/>.

3. Voir en ligne : <https://www.ameli.fr/>.

4. Voir en ligne : <https://www.facebook.com/medecinssansfrontieres/>.

5. Voir en ligne : <https://www.facebook.com/medecinsdefrance/>.

6. Voir en ligne $:<$ https://www.clubdesmedecinsblogueurs.com/>.

7. Voir en ligne : <http://armance.overblog.com/>.

8. Voir en ligne :

<https://www.youtube.com/channel/UCR0YcNmQbNNFYe2Onk4Ox5g>.

9. Voir en ligne :

<https://www.youtube.com/channel/UCtNyuN_HFINYO0-gqXwhLTg>.

10. Voir en ligne :

<https://www.youtube.com/channel/UCGmofCPILo0W4L7HNfrHgEg>.

11. Voir en ligne : <http://www.infirmiers.com/forum/ordre-infirmier.html>.

12. Voir en ligne $:<$ https://www.groupeprofessionsante.fr/le-groupe>.

13. Voir en ligne : <https://www.lequotidiendumedecin.fr/>. 
Pharmacien $^{14}$, Le Généraliste ${ }^{15}$, etc.) visant à communiquer des informations fiables au sein du monde médical. L'appartenance d'infirmiers.com à ce groupe médiatique garantit à la fois la stabilité de sa présence sur Internet et la fiabilité des informations échangées. De même, ce site est marqué par son forum regroupant jusqu'à mars 2020 plus de 316600 membres intéressés à échanger sur l'actualité infirmière. Enfin, nous avons remarqué le dynamisme communicatif de ce réseau numérique à tel point que nous avons recensé (mars 2020), par exemple, plus de 1003500 messages produits dans une rubrique intitulée Admission en IFSI. Il est à noter que ce forum se divise en trois sous-forums dont chacun traite des thèmes bien précis : forums généraux (aides-soignants, concours infirmiers, profession infirmière, etc.), forums spécialisés (infirmiers de bloc opératoire, cadres de santé, infirmiers anesthésistes, etc.) et Tribune/Divers (petites annonces, co-voiturage, etc.). Dans ces échanges numériques actifs, le locuteur tente de « construire » les différents aspects de son identité au sein de cette communauté de pratique numérique.

Quant à la méthodologie de recherche suivie, notre étude adopte une approche écologique du discours numérique (Paveau 2017) qui permet de prendre en considération non seulement les locuteurs et leurs échanges discursifs mais aussi l'environnement numérique dans lequel ces derniers se produisent. Face à la complexité de l'objet d'étude, il nous semble pertinent de mener une étude exploratoire qui met en lumière l'analyse de contenu (discursif et formatif) de certains exemples (messages, pseudonymes, éléments visuels et stratégies discursives). En raison du grand nombre de messages produits, nous optons plutôt pour une analyse qualitative ayant pour objectif de souligner les principales caractéristiques récurrentes de l'identité numérique du locuteur.

\section{L’identité sociale du locuteur professionnel dans le forum infirmiers.com}

Dès son arrivée sur le forum, le locuteur est amené à élaborer son identité pour devenir membre de cette communauté professionnelle numérique. Il s'agit de l'ethos montré qui indique l'appartenance du locuteur à la communauté professionnelle en question. Il a alors trois possibilités. Soit il reproduit son identité réelle de la vie sociale (nom, prénom, âge, etc.), soit il crée une nouvelle identité numérique qui correspond mieux à ses besoins personnels et ses intentions professionnelles, soit il peut

14. Voir en ligne : <https://www.lequotidiendupharmacien.fr/>.

15. Voir en ligne : <https://www.legeneraliste.fr/>. 
faire un mélange de ces deux identités pour créer une nouvelle identité numérique dite métisse. On cite l'exemple suivant :

Figure 1 : Exemple de l'identité sociale du locuteur sur le forum infirmiers.com

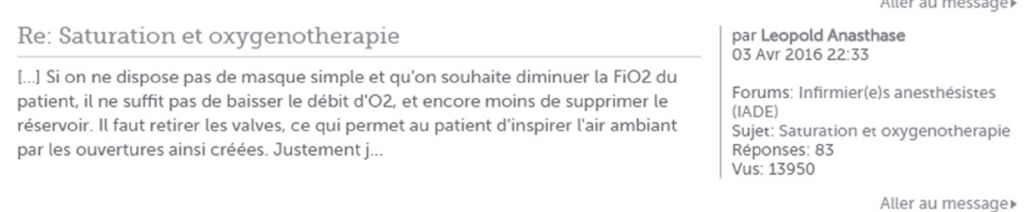

Cet exemple souligne les deux composantes de l'identité sociale du locuteur qui comprend les éléments suivants :

- l'identité déclarative : le pseudo du participant (Leopold Anasthase), la date et l'heure du message (3 avril 2016/22h33), l'intitulé du sous-forum (infirmiers anesthésistes) et le sujet abordé (saturation et oxygenothérapie) ;

- l'identité calculée : le nombre de réponses au message (83) et le nombre de consultations (13 950).

Dans le cadre de l'analyse de l'identité sociale, on accorde un intérêt particulier aux pseudonymes, éléments visuels utilisés et relations interpersonnelles :

\section{1. Les pseudonymes du locuteur}

Lors de notre étude de ce forum professionnel, on remarque que le locuteur se présente d'abord grâce à un pseudonyme qui constitue un trait sociolinguistique reflétant parfois le caractère de son porteur. "C'est le “je” qui se nomme et non l'autre qui nomme le “je” (Martin 2012:57). Selon la même auteure, il s'agit d'un " nom fait à plaisir ", soit pour masquer la vraie identité du locuteur, soit pour mettre en lumière un aspect particulier (personnel, professionnel, etc.). (Paveau 2012b), quant à elle, souligne cette relation de pérennité entre le locuteur et son pseudonyme dans la mesure où il le choisit et y reste fidèle dans la plupart des cas. "Après analyse des pseudonymes [...] les conclusions montraient que le "nom fait à plaisir" prenait ses sources dans le paradigme de l'espace privé du sujet social » (Martin 2012 : 93). Le pseudonyme pourrait avoir une dimension sémantique qui permet de dévoiler une "facette " de soi en ligne (Cislaru 2009). Dans le corpus étudié, on observe que les locuteurs adoptent trois formes principales de pseudonymes exprimant plusieurs aspects de leur identité sociale. La première concerne 
le recours aux éléments langagiers (souvent des prénoms) accompagnés des chiffres (julie60400, Laetitia13340, lilidu61, Audrey11, etc.) qu'on pourrait interpréter selon les hypothèses suivantes : date de naissance, âge, numéro de son département, date de l'inscription au forum, etc. Citons les cas suivants de certains locuteurs qui utilisent des pseudonymes composés d'un prénom suivi du code de leurs départements :

Figure 2 : Exemples de certains pseudonymes marquant la localisation géographique de leurs locuteurs
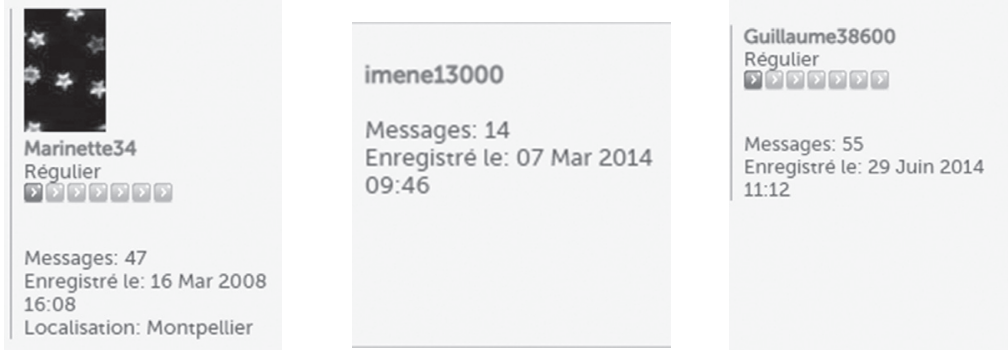

Dans la deuxième forme du pseudonyme, on constate la présence des éléments langagiers auxquels le locuteur ajoute des signes typographiques (ex : $€, \ldots,-, @$, etc.) comme dans les exemples suivants : @tchoum, $M @ N @$, S@ndy, etc. Une telle forme adoptée montre l'impact du support numérique sur le choix de pseudonyme en vue de marquer sa différence par rapport aux autres locuteurs professionnels. Dans la troisième forme, les locuteurs choisissent des noms communs (ex : neigeeternelle, Escargot Bleu, l'agitateur, madouce, etc.) visant à se distinguer par rapport aux autres participants comme c'est le cas des exemples suivants :

Figure 3 : Exemples de certains locuteurs utilisant des noms communs
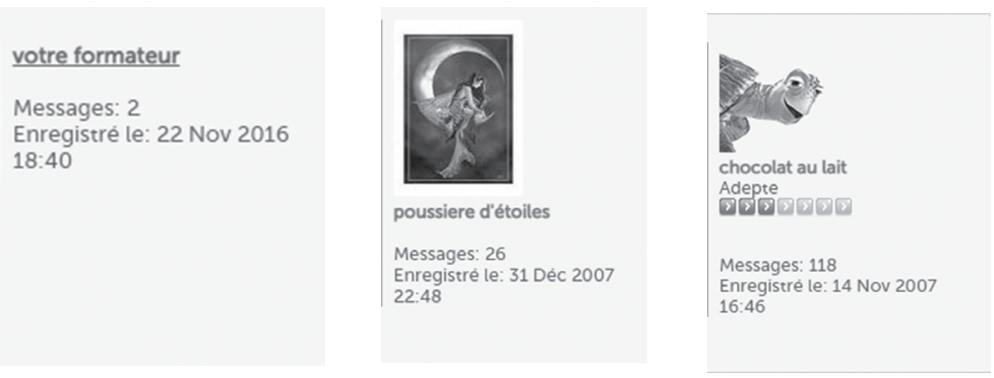
D'autres locuteurs font une rétrospection en soulignant un aspect personnel auquel ils s'attachent pour s'en créer une nouvelle identité. Celle-ci pourrait être parfois le résultat d'un métissage linguistique regroupant deux ou plusieurs langues dans le même pseudonyme formé (ex : MademoiselleJames, calimero punk des alpes, etc.). Un tel phénomène pourrait être justifié par le fait qu'internet favorise le contact des langues dans la mesure où les internautes sont souvent exposés à plusieurs langues lors de leurs activités numériques. Dans certains cas, des participants s'intéressent à élaborer leur identité sociale en ayant recours à des pseudonymes empruntés à l'anglais (WALTER, NURSE1, Coshka, elynn-the-dark-angel, Keep-the-smile, Smile, Juste-Tite-Miss, etc.). Ces emprunts reflètent le contexte numérique mondialisé dominé par la langue de Shakespeare qui est également utilisée dans quelques activités professionnelles médicales (diagnostique, médicaments, documentation, conférences, etc.).

Certains participants utilisent des noms propres (Jean-Ralphio, Laura, Daphnée, etc.) tandis que d'autres optent pour des combinaisons socioprofessionnelles regroupant à la fois la dimension professionnelle (profession, statut, etc.) et la dimension personnelle (prénom ou localisation géographique) : psychatrik angel, inf36-87, zarainfirmière, infirmièrececile, infirmièreencolère, Kine83260, spécialiste retraité, etc. Dans ces derniers exemples, le choix d'un pseudonyme professionnel permet de marquer l'appartenance à cette communauté de pratique et d'attribuer au sujet-parlant une certaine légitimité de parole appelée aussi le droit de parole (Charaudeau 2009) au sein de ce groupe professionnel numérique. Il s'agit également d'une stratégie discursive qui donne la possibilité au locuteur d'être reconnu par ses pairs pour les préparer à adopter son discours. Enfin, notre analyse souligne que la dimension professionnelle occupe le devant de la scène dans le choix des pseudonymes dans la mesure où le locuteur s'attache à utiliser certains termes issus de son milieu professionnel : lieux (clinique, labo, etc.), spécialités (L'ambulancier, paramédic86, samulorraine, etc.), outils (seringa, stéthoscope, etc.), maladies (tetanos, etc.) et organes (cortex, etc.), etc. Cette omniprésence terminologique médicale pourrait être justifiée par la nature de ce forum créé essentiellement pour répondre à des besoins professionnels.

\section{2. Les éléments visuels de l'identité sociale}

Grâce aux potentialités du numérique, le locuteur professionnel a recours à des éléments visuels contribuant à marquer certains aspects particuliers ayant pour objectif d'affirmer les éléments qui suivent. 


\section{2. 1. Dimension professionnelle omniprésente}

Il s'agit souvent de mettre des images ou des logos qui indiquent l'appartenance du locuteur au monde médical. Pour réussir son discours, le participant doit afficher ses qualités professionnelles "sur un mode généralement implicite "(Kerbrat-Orecchioni 2002 : 42). C’est le cas des exemples suivants où les locutrices ont choisi de mettre une image d'infirmière :

Figure 4 : L'appartenance professionnelle des locuteurs au monde médical
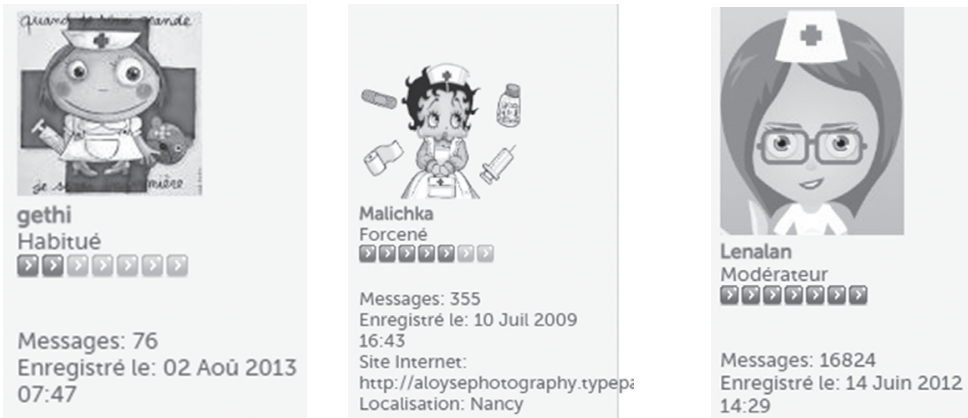

\section{2. 2. Préférences personnelles}

Certains locuteurs mettent des images exprimant leurs préférences personnelles (animaux, équipes, chanteurs, nature, etc.) qui montrent leur impact sur l'identité du locuteur numérique. Un tel procédé favorise ainsi la réalisation d'un fusionnement identitaire du social et du professionnel dans cet espace technodiscursif. On peut aussi émettre une autre hypothèse selon laquelle ces locuteurs veulent protéger leur propre identité privée en choisissant des images stockées ou trouvées sur Internet :

Figure 5 : Des exemples montrant les préférences personnelles des locuteurs professionnels
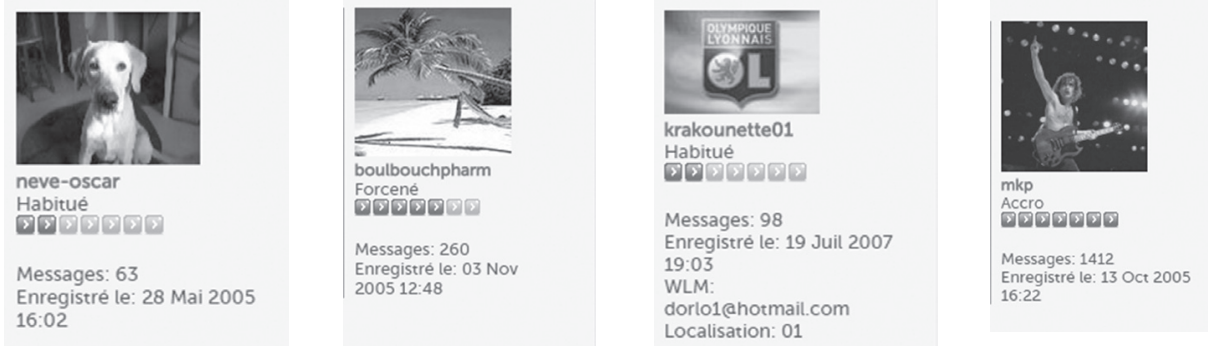
5. 2. 3. Appartenance à une institution professionnelle donnée

D'autres locuteurs préfèrent affirmer leur appartenance à un milieu professionnel particulier en précisant leur lieu de travail. Il s'agit alors d'un acte ayant pour objectif de légitimer le discours numérique en " s'octroyant une position institutionnelle et marquant son rapport à un savoir" (Amossy 2002 : 239). C'est le cas des exemples présentés ci-dessous :

Figure 6 : L'appartenance géographique des locuteurs à un milieu médical précis
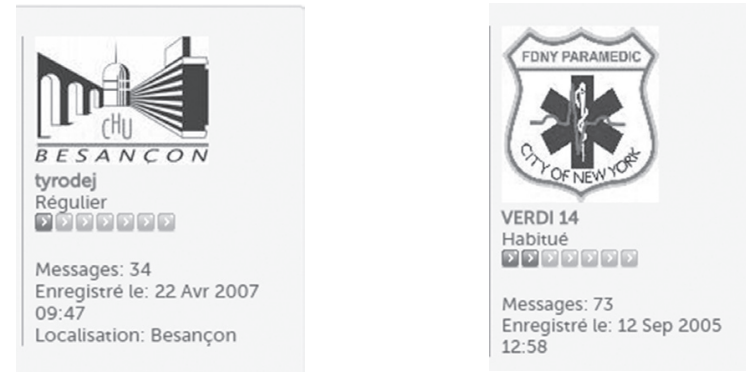

\section{2. 4. Identité privée}

Pour confirmer leur identité privée dans les échanges numériques, certains participants n'hésitent pas à mettre leurs photos personnelles qui indiquent la part importante du social dans ces échanges professionnels :

Figure 7 : Des locuteurs affirmant leur identité personnelle grâce à des photos personnelles
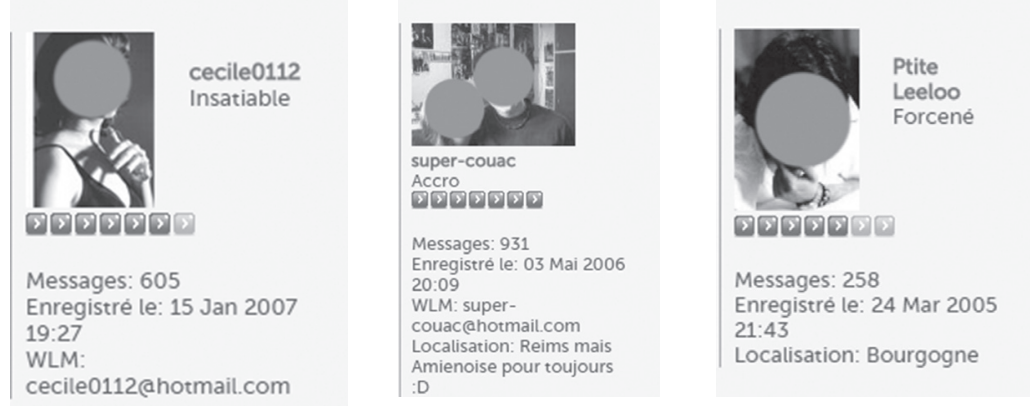


\section{2. 5. Présence des émojis}

Les émojis consistent à utiliser des codes représentant un petit visage créé à l'aide de caractères de ponctuation. Ils ont pour objectif d'exprimer l'état d'esprit du locuteur tout en donnant une dimension personnelle de son discours. Ces emojis peuvent être de type expressif, interprétatif, relationnel et procédé de politesse (Marccocia 2002). Dans le forum d'infirmiers.com, on remarque que les locuteurs ont recours de temps en temps à ces éléments visuels pour créer une ambiance conviviale notamment dans leurs échanges professionnels. C'est le cas du passage suivant tiré du sous-forum «Phrases cultes des patients » où une locutrice raconte une situation lors de sa carrière professionnelle : " une mamie qui me propose un gâteau. Je refuse poliment et elle insiste. Je lui dis non merci je fais un régime et là elle me regarde "ba il est temps ! $\Theta \triangleq($ akselle, 05/12/2003) Dans l'exemple précédent, on constate que l'utilisation de l'émoji permet d'exprimer la réaction amusante de la locutrice, réalisant ainsi une complémentarité informative entre le textuel et le visuel dans ce message. Tous ces éléments visuels rendent le discours numérique professionnel à la fois riche et varié aux niveaux informationnel, argumentatif et interactionnel.

\section{3. Les relations interpersonnelles au sein de cette communauté professionnelle numérique}

Pour mieux développer l'identité sociale de ses participants, le forum analysé leur propose une rubrique pour se présenter tout en accordant un intérêt spécial à leurs carrières professionnelles. Certes, il s'agit d'un forum professionnel mais ce dernier est avant tout un réseau social facilitant au locuteur de tisser des relations interpersonnelles au sein de sa communauté de pratique. Voici les exemples suivants :c

- «Promo 2001/2004, IFSI théodore Simon (93) je bosse depuis un mois en chirurgie viscérale à bry sur marne $(94)$...heureuse $"(c y l$, 21/03/2005);

- "promo 2004/2007, ifsi cochin, 110 élèves au départ, plus que 100 " (flam3, 22/03/2005);

- " aide soignante diplômée en 94, 2 ans auprès d'ado et d'adulte hanidicapés (pendant le CAFAS) une pose d'un an et demi pour m'occuper de ma fille, 1997-2001 as dans une MAS arrêt maladie maternité, congé maternité, congé parental pour mon fils et pendant ce temps prepa (2004) au concours d'infirmière 2004-2007 ifsi avec 94 esi » (Flossy, 21/03/2005). 
Ce réseau numérique professionnel incite également ses participants à aborder des thèmes variés de leur choix en créant un sous-forum intitulé " La libre tribune d'Infirmiers.com ». On peut y trouver certains échanges qui ne sont pas forcément liés au milieu médical tels que « Présidentielles 2017 : quelles sont vos attentes ? ", "Qu'est-ce que vous écoutez en ce moment ? ", "Faut-il supprimer le Sénat ? ", " Pour les célibataires ", etc. Ainsi, cet espace social a pour but de favoriser les relations interpersonnelles au sein de cette communauté professionnelle numérique, donnant la liberté aux locuteurs d'aborder les sujets qui les intéressent. D'où le fusionnement discursif du social et du professionnel dans les échanges produits comme le montre le passage suivant tiré du sous-forum intitulé « Pour les célibataires » : «Moi je suis celib, ...Depuis un an.. Et ça me saoule !!! Je cherche, je cherche... Vous inquiétez pas, j'ai des propositions mais je veux plus me planter (...) Bisous et souhaitez moi bonne chance dans la quête de mon futur cher et tendre !!! » (Aramis, 29/11/2003). Pour favoriser les dimensions conviviale et interpersonnelle au sein de cette communauté professionnelle, le modérateur de ce forum propose aussi aux participants un autre sous-forum intitulé "Votre Date d'Anniversaire " ayant pour but de favoriser la dimension sociale dans les interactions produites. Voici certains messages échangés à cette occasion : " Joyeux anniversaire SAGELINE et plein de bonheur dans ta vie ", "Joyeux anniversaire Laury, Que du bonheur pour toi et surtout une santé de fer ", "Passe une agréable journée " et "Joyeux anniv MA Laury ». Grâce à ces différents procédés discursifs (textuels et visuels), les locuteurs sont en mesure de mieux développer leur identité sociale notamment en relation avec leurs carrières professionnelles. Ainsi, on assiste à un fusionnement informatif entre les données personnelles, les pseudonymes et les éléments visuels sélectionnés, renforçant l'identité sociale au sein de ce forum professionnel en ligne.

\section{L'identité discursive des locuteurs professionnels en ligne}

La construction de l'identité sociale ne fait que préparer le terrain au locuteur pour sa prise de parole au sein de cet espace professionnel en ligne. Alors, il est censé répondre à la question suivante : " Je suis là pour quoi faire, en fonction du statut et du rôle qui m'est assigné par la situation ? " Dans ce contexte, il nous parait intéressant de mettre en relief la notion de pratique langagière conçue comme une pratique sociale où les mots et les discours " ne se contentent pas de refléter le social, ils en sont partie prenante et ils agissent sur le social, y produisant des effets spécifiques que l'analyste doit repérer [...]. Tout acte, qu'il soit technique 
ou pas est conçu, représenté par des acteurs sociaux, c'est-à-dire travaillé dans le matériau sémiotique qu’est le langage " (Boutet 1985 : 6). Dans le cadre de ce discours numérique professionnel, le locuteur est en mesure d'adopter plusieurs stratégies en vue d'élaborer son identité discursive dans ses échanges avec ses pairs.

D'abord, le locuteur pourrait avoir une attitude de neutralité marquée par l'effacement de tout jugement personnel pour se présenter en tant que témoin qui rapporte ce qu'il a déjà acquis dans son domaine professionnel. On constate cette stratégie discursive dans l'exemple suivant où le locuteur indique la valeur d'un master intitulé Cadres $d u$ secteur sanitaire et social: "Un master "seul" ne permet pas de travailler dans le public, qui a ses propres filières (IFCS, EHESP....), il sert de base pour préparer les concours publics (EHESP) afin de devenir DESSMS, DH, AAH...» (Jo_bis, 20/02/2016). Dans le message précédent, on constate l'absence totale du locuteur dans les énoncés produits où il se contente de fournir les informations nécessaires sans donner aucun jugement personnel de sa part. Cette neutralité discursive reflète une objectivité informative visant à indiquer les différentes spécificités du master en question.

De même, le locuteur peut adopter l'attitude de distanciation (Vion 2005) qui lui donne la possibilité de s'exprimer en tant qu'expert du domaine concerné. Pour ce faire, il a recours à ses connaissances et ses expériences professionnelles en vue de juger, commenter, analyser et expliquer des faits du monde médical. Dans ce contexte, c'est l'identité sociale du locuteur qui justifie sa prise de parole tout en lui attribuant une certaine autorité discursive au sein de la communauté numérique professionnelle. En analysant certains messages, on constate cette attitude discursive où le locuteur vient en aide à ses pairs pour répondre à leurs questions en tant que spécialiste du sujet abordé. Dans le passage suivant, le locuteur se charge de fournir des informations sur la composition de SAP (Seringue Auto-Pousseuse) : « 2 gammas $/ \mathrm{kg} / \mathrm{min}$, ça fait 7200 gammas/h $(2 \times 60 \times 60)$. Pour avoir $8 \mathrm{mg} / \mathrm{mL}$, on prend une ampoule de $200 \mathrm{mg}$ que l'on complète jusqu'à $25 \mathrm{~mL}$, soit 8000 gammas/ML. "(Pilou-Pilouuu, 12/11/2015) Au niveau lexical, on remarque que le locuteur utilise un technolecte particulier (Lerat 1994) facilitant les échanges au sein de la communauté médicale. À partir de son expérience scientifique, il précise les étapes à suivre pour composer le produit médical concerné, marquant une distanciation discursive qui vise à attribuer une certaine crédibilité scientifique à son discours professionnel numérique. 


\section{I42 / HANI QOTB}

Dans d'autres cas, le locuteur pourrait manifester un engagement discursif qui consolide sa prise de position à propos du sujet abordé avec ses pairs. Cette attitude discursive consiste à " construire l'image d'un sujet parlant "être de conviction". La vérité ici, se confond avec la force de conviction de celui qui parle, et celle-ci est censée influencer l'interlocuteur " (Charaudeau 2009 : 5). Ainsi, le locuteur met en place une stratégie argumentative contribuant à convaincre ses interlocuteurs d'adopter son point de vue. Pour ce faire, il avance tous les arguments scientifiques pour défendre le bien-fondé de sa position. On cite l'exemple suivant sur le calcul d'une dose de dopamine où le locuteur professionnel présente ses arguments face à une démarche qualifiée d' "inadaptée » :

« Cet exercice est inadapté :

- On ne doit plus parler de "gamma «; pour ceux qui s'en souviennent, gama la lessive poids lourd ; il s'agit de microgramme par kilogramme de poids corporel et par minute, ou $\mu \mathrm{g} / \mathrm{kg} / \mathrm{min}$;

- la dopamine n'est plus utilisée à cette dose (et d'ailleurs est très utilisée actuellement chez l'adulte);

- la concentration est trop élevée et conduit à un débit trop faible (sauf peut-être en néonatalogie, mais avec un poids corporel de $60 \mathrm{~kg}$, ça serait un beau-bébé ) » (Léopold Anasthase, 13/01/2016)

Certains locuteurs ont également recours à plusieurs attitudes discursives dans la même production langagière pour adopter un discours démonstratif. Ce dernier consiste non seulement à fournir des arguments mais aussi à suivre un mode de raisonnement ayant pour objectif d'accepter le point de vue présenté comme une vérité incontournable à laquelle tout un chacun doit se soumettre. Pour ce faire, le locuteur peut faire appel à la tactique discursive de captation en adoptant certaines techniques : abondance des informations, arguments scientifiques, avis professionnels, mise en relief des informations principales, images, graphes, etc. Ces éléments constituent une démonstration discursive permettant au locuteur d'être cru par ses pairs. Dans un sous-forum intitulé "Les IADE : votre secret pour trouver les veines ? ", un locuteur explique à ses pairs les différentes techniques nécessaires à suivre pour le patient (Revenge, 17/07/2005). Sa présentation professionnelle semble être convaincante pour ses pairs dans la mesure où ces derniers épousent son point de vue en le remerciant de 
son explication : "Merci pour vos précieux conseils », "Merci Revenge pour ce beau résumé » et " Bravo, Bravo».

\section{Conclusion}

Dans cet article, nous avons tenté de comprendre les différentes spécificités de l'identité du locuteur professionnel en ligne notamment dans les forums médicaux. D’après l'analyse de différents échanges, l'identité numérique du locuteur professionnel s'est avérée complexe dans la mesure où elle est marquée tantôt par une articulation tantôt par une tension entre ses deux dimensions sociale et professionnelle, réalisant un certain fusionnement entre les deux sphères identitaires. Ainsi, le locuteur professionnel est amené à élaborer soigneusement son identité qui constitue à la fois une condition préalable et une partie intégrante de sa participation sur les réseaux sociaux professionnels. Au niveau de son identité sociale, le sujet-parlant accorde une importance particulière à affirmer son appartenance au milieu professionnel concerné en vue de justifier sa présence au sein de ce réseau social spécialisé (choix de son pseudonyme, mise en relief de son statut professionnel, utilisation des termes techniques, etc.). De même, le dispositif technodiscursif du forum contribue à mieux développer l'identité professionnelle en ajoutant des éléments visuels pour renforcer sa légitimité dans ces échanges spécialisés. Quant à l'identité discursive, le locuteur professionnel peut avoir recours à plusieurs stratégies (neutralité, distanciation, engagement et démonstration) pour que son discours soit reconnu par sa communauté de pratique en ligne.

Au gré des échanges au sein de ces réseaux professionnels en ligne, on assiste à l'émergence d'une identité numérique collective qui représente l'ensemble des caractéristiques sociales et discursives communes au sein de la même communauté de pratique. Certes, chaque locuteur a ses spécificités personnelles mais il partage aussi des intérêts professionnels et des stratégies discursives avec ses pairs. Ces derniers ont recours à des usages lexicaux et discursifs propres à leur domaine de spécialité, formant un technolecte au sein de leur communauté professionnelle. Il nous semble également nécessaire de souligner les relations réciproques entre l'identité individuelle du locuteur et l'identité collective de sa communauté de pratique dans la mesure où l'une a des répercussions sur l'autre à condition que celle-ci continue à être active et dynamique sur Internet. Reste à prendre en compte la dimension hybride (sociale et professionnelle) des échanges discursifs favorisée par le statut non institutionnel du forum qui laisse le champ libre aux locuteurs d'aborder des thèmes relatifs à leurs sphères professionnelle, sociale et personnelle. 
I44 / HANI QOTB

\section{Bibliographie}

Amossy R. (2002), "Ethos », dans Chteau P. \& Maingueneau D. (dir.), Dictionnaire d'Analyse du Discours, Paris, Seuil, p. 238-240.

Boutet J. (1985), Construction sociale du sens dans des entretiens d'ouvrières et d'ouvriers. Paris, ministère des Droits de la femme, ronéo.

Breslin J., Passant A. \& Decker S. (2009), The Social Semantic Web, Berlin/ Heidelberg, Springer.

Bruillard M. (2006), "Le forum de discussion : un cas d'école pour les recherches en EIAH », STICEF 13. En ligne : <http://sticef.univ-lemans.fr/num/vol2006/sticef_2006_ns_edito.htm>.

Charaudeau P. (2009), « Identité sociale et identité discursive. Un jeu de miroir fondateur de l'activité langagière ", dans Charaudeau P. (dir.), Identités sociales et discursives du sujet parlant, Paris, L'Harmattan.

Charaudeau, P. (2005), Le discours politique. Les masques du pouvoir, Paris, Vuibert.

Cislaru G. (2009), " Le pseudonyme, nom ou discours ? ", Les Carnets du Cediscor 11. En ligne : <http://cediscor.revues.org/746>.

Georges F. (2009), "Représentation de soi et identité numérique : une approche sémiotique et quantitative de l'emprise culturelle du Web 2.0. », Réseaux 154, p. 165-193.

Iteanu O. (2008), Lidentité numérique en question, Paris, Eyrolles.

Kerbrat-Orecchioni C. (2002), "Système linguistique et ethos communicatif ", Cahiers de praxématique 38, p. 35-57.

Le Journal Officiel (1999), 16 mars.

Lerat P. (1994), Les langues spécialisées, Paris, PUF.

Licoppe C. (2009) (dir), L'évolution des cultures numériques. De la mutation du lien social à l'organisation du travail, Paris, FYP Éditions.

Loisy C., Mailles-Viard Metz S. \& Benech P. (2010), "Scénarios pour l'identité numérique et la construction de l'orientation ", Journées Communication et Apprentissage Instrumentés en Réseau. En ligne : $<$ http://halshs.archives-ouvertes.fr/halshs-00529462/fr/>. 
Maingueneau D. (2002), «L'ethos, de la rhétorique à l'analyse du discours », version raccourcie et légèrement modifiée de "Problème d'ethos", Pratiques 113-114. En ligne : <http://dominique.maingueneau.pagesperso-orange.fr/intro_company.html>.

Mangenot F. (2002), "Forums et formation à distance : une étude de cas ", Éducation permanente 152, p. 109-119.

Marccocia M. (2002), «Les communautés en ligne comme communautés de paroles », Journées d'études "Internet, jeu et socialisation », 5-6 décembre, ENST Paris. En ligne : <http://www.instituttelecom.fr/ archive/156/ActesMarcoccia.pdf>.

Martin M. (2012), Se nommer pour exister. L'exemple du pseudonyme sur l'internet, Paris, L'Harmattan.

Paveau M.-A. (20122012a), « Réalité et discursivité. D’autres dimensions pour la théorie du discours ", Semen, p. 95-115.

Paveau M.-A. (2012b), "Les écritures de Protée : identités pseudonymes ", La pensée du discours. En ligne : <http://penseedudiscours.hypotheses. org/?p=10057>.

Paveau, M.-A.(2017), L'Analyse du discours numérique. Dictionnaire des formes et des pratiques, Paris, Hermann.

Vion R. (2005), "Séquentialité, interactivité et instabilité énonciative ", Cahiers de praxématique 45, p. 25-50.

\section{Sitographie ${ }^{16}$}

https://solidarites-sante.gouv.fr/

http://www.santepubliquefrance.fr/

https://www.ameli.fr/

https://www.facebook.com/medecinssansfrontieres/

https://www.facebook.com/medecinsdefrance/

https://www.clubdesmedecinsblogueurs.com/

http://armance.overblog.com/

https://www.youtube.com/channel/UCR0YcNmQbNNFYe2Onk4Ox5g

https://www.youtube.com/channel/UCtNyuN_HFINYO0-gqXwhLTg

16. La date de la dernière consultation des sites cités est le 03/03/2020. 


\section{I46 / HANI QOTB}

https://www.youtube.com/channel/UCGmofCPILo0W4L7HNfrHgEg http://www.infirmiers.com/forum/ordre-infirmier.html https://www.groupeprofessionsante.fr/le-groupe https://www.lequotidiendumedecin.fr/ https://www.lequotidiendupharmacien.fr/ https://www.legeneraliste.fr/

Article reçu en septembre 2019. Révision acceptée en mars 2020. 